\title{
THE DIGITALIZATION OF ANCIENT EGYPTIAN COFFINS: A DISCUSSION OVER DIFFERENT TECHNIQUES FOR RECORDING FINE DETAILS
}

\author{
A. Mandelli ${ }^{1, *}$, L. Perfetti ${ }^{1}$, F. Fiorillo ${ }^{1}$, F. Fassi ${ }^{1}$, C. Rossi ${ }^{2}$, C. Greco ${ }^{3}$ \\ 1 3D Survey Group, Politecnico di Milano, Dept. of Architecture, Built environment and Construction engineering (ABC), Italy - \\ (alessandro.mandelli, luca.perfetti, fausta.fiorillo, francesco.fassi)@polimi.it \\ ${ }^{2}$ Politecnico di Milano, Dept. of Architecture, Built environment and Construction engineering (ABC), Italy - \\ corinna.rossi@polimi.it \\ ${ }^{3}$ Museo Egizio, Turin, Italy - christian.greco@museoegizio.it
}

\author{
Commission II, WG II/8
}

KEY WORDS: Egyptian coffin, structured-light scanner, photogrammetry, 3D documentation, micro-mapping

\begin{abstract}
:
This work starts from the request to have a physical high-resolution 3D model of the external, anthropoid coffin of the scribe Butehamon, held at the Museo Egizio, Turin. At the time of writing, a replica of the coffin, based on this survey work, functions as final and focal installation of the temporary exhibition Archeologia Invisibile of the Museo Egizio, Turin, running from March 2019 to January 2020. The replica acts as support for a micro-mapping installation meant to re-project a pattern of images onto the coffin's surface, including the results of different radiometric and colourimetric analyses performed in the recent past by Museo Egizio and Musei Vaticani. This collaborative work encouraged a thorough discussion on the interaction between scientists and humanists engaged in the study of archaeological finds, on the needs and expectations of both sides, and on the technical problems relating to handling objects of different sizes.
\end{abstract}

\section{FRAMEWORK}

Today we are immersed in what is called the digital revolution, which is deeply transforming our cognitive approach and working methods. In archaeology, laser scanning, photogrammetry and 3D modelling enable archaeologists to document the whole excavation process and reconstruct contexts even after they have been physically removed. We can reproduce a coffin with sub-millimetric accuracy and record all its phases of production and reuse. Non-invasive diagnostic imaging enables us to peer inside a still sealed vessel and virtually unwrap mummies. Accurate analyses now give scholars the opportunity to observe the fibres of a papyrus, helping them recomposing ancient documents. Finally, digital communication enables us to create virtual working environments in which scholars from all over the world can confer and compare their data. This does not mean that the humanists' role is becoming secondary. Actually, it is quite the contrary: the data that we are nowadays able to collect are increasingly detailed and complex, and require an equally increasingly high level of interpretation. Scientists and humanists have to work together even more closely to unravel the complexity of the contemporary world (Greco, 2019, Rossi, 2019). The scientist/technicians must, on their side, define guidelines and approaches to survey and deliver the complete three-dimensional digital model of artefacts (Barsanti et al., 2014). The definition of a reference protocol passes through the identification of methodological phases regarding the 3D survey:

- design of the survey: searching for the best instruments, definition of position and number of stations, use of targets, desired resolution, final accuracy of the model;

- data acquisition;

- data integration: direct, total station, detailed surveys;

- data elaboration;

- data delivery: 3D models, 2d graphic vector or raster representation, geometric comparisons.
In this specific case, the goal of the survey was the construction of a 3D high-resolution representation of an anthropoid coffin, able to describe every single fine detail. The expected result would be useful both for diagnostic, study and preservation aims, as well as for dissemination purposes (Guidi et al., 2004, Tucci et al., 2017), recently greatly enhanced by the advancement of technology (Fischnaller et al., 2015). The survey of this coffin thus offered the chance to discuss the connection between expectations and workflow from zero, and experiment in various directions. Two different techniques were adopted and combined: the survey with a triangulation scanner, useful to record all the small decorations of the surface (Taylor et al., 2002), and the photogrammetric approach, able to obtain both a complete 3D model and a high-quality and sub-millimetric texture of the object.

\section{THE COFFIN}

The scribe Butheamon was buried into two coffins, placed one into the other; the survey work concerned both parts (lid and case) of the larger, outer coffin (Figure 1). In this article, we present the workflow to record and elaborate in particular the lid of the coffin - a procedure that can be replicated for other parts. The dimensions of the coffin as a whole are $211 \mathrm{~cm}$ (length), 74 $\mathrm{cm}$ (width), and $43 \mathrm{~cm}$ (depth). The importance of the coffin is related to both the characteristics of the coffin, belonging to the Third Intermediate Period, as well as to the role of Butehamon during his life: he was the descendent of an important dynasty of scribes, and held a key role in the artisans' community of Deirel-Medina, in charge of building the pharaohs' tombs in the Valley of the Kings and of the Queens. The artefact arrived in Turin in 1824 with the Drovetti collection and it was probably found in a re-used tomb of a dignitary named Nakhtmin (museivaticani.va). Nowadays, the coffins of Butehamon are on display in the Galleria dei Sarcofagi at Museo Egizio, Turin.

\footnotetext{
${ }^{*}$ Corresponding author
} 


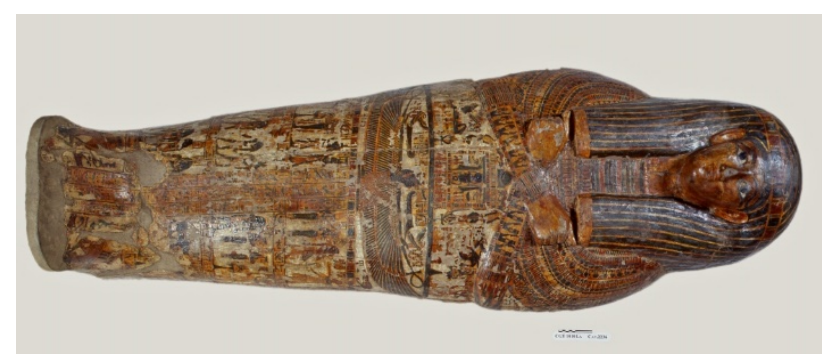

Figure 1. The external coffin of Butehamon, from the catalogue of the Museo Egizio, Turin.

The non-invasive diagnostic campaign, carried out in collaboration by Museo Egizio and Musei Vaticani, highlighted interesting clues about the history of the artefact and about the carpentry techniques which were used (Prestipino et al., 2015). The x-rays revealed the general structure of the lid (made of 16 elements) and the lower part (made of 11 elements), confirming that only the elements of the latter were constructed for Butehamon, while the others were obtained by re-using parts of at least four different coffins. Moreover, the x-rays showed several interventions to re-shape the reused elements, both in the area of the hands and of the face. The direct observation of the layer of paint, along with the microstratigraphy analyses, revealed two different, overlapping painting layers. On the right side and on the head these two layers are preceded by a third one, more ancient, which belonged to a black painted coffin.

\section{THE SURVEY}

\subsection{The lid of the coffin}

The peculiarity of the coffin is that both sides (interior and exterior) are completely covered by detailed decorations, and therefore the survey has to record precisely both the geometry and the colour. A full-frame camera was used to this purpose; a triangulation scanner, instead, was used to acquire only the shape, in order to use the geometry as a benchmark for the photogrammetric method (Pontin et al., 2005).

This workflow derives from previous tests conducted on other heritage artefacts, such as the Pietà Rondanini by Michelangelo (Mandelli et al., 2016), improved thanks to the use of flashes and polarized light during the photogrammetric acquisition.

The results of the aforementioned case study and the literature of recent years, state that photogrammetry, in specific conditions, grants outcomes of comparable or even higher resolution than the triangulation scanners. However, the accuracy of the photogrammetric 3D models depends on many different factors, such as GSD (Ground Sampling Distance), network geometry, texture quality, illumination conditions and more, that can be challenging to control. That is the reason why, in this specific occasion in which we expected to extract sub-millimetric details from the photogrammetric approach, we chose to use the scanner model as reference. The uncertainty about the accuracy is also linked to the shiny character of the paintings decorating the entire coffin. The darkest areas, brown and black, could produce a thin layer of noise around the real surface of the object, preventing the correct description of the coffin's shape.

In most cases, the photogrammetric approach appears to be more convenient, because of its versatility, portability and lower cost in comparison with a scanner; least, but not last, because it provides complete data, including not only shape but also colour (Gomes et al., 2014). This last aspect is strongly felt in the archaeological field: archaeological fieldwork is a form of destruction of the site, as it proceeds by clearing away objects and contexts, in order to gradually uncover the underlying levels and follow the thread of history backwards. Thanks to photogrammetry, first the images and then the 3D models become, in turn, reliable and realistic archives of data.

The survey phase lasted four days during which two surveyors applied in parallel, in different rooms, both the scanner and the photogrammetric survey techniques. The room dedicated to photogrammetry was restricted to the public, and was prepared to guarantee optimal light control of the environment: there were no reflections, refractions and shades (Figure 2).

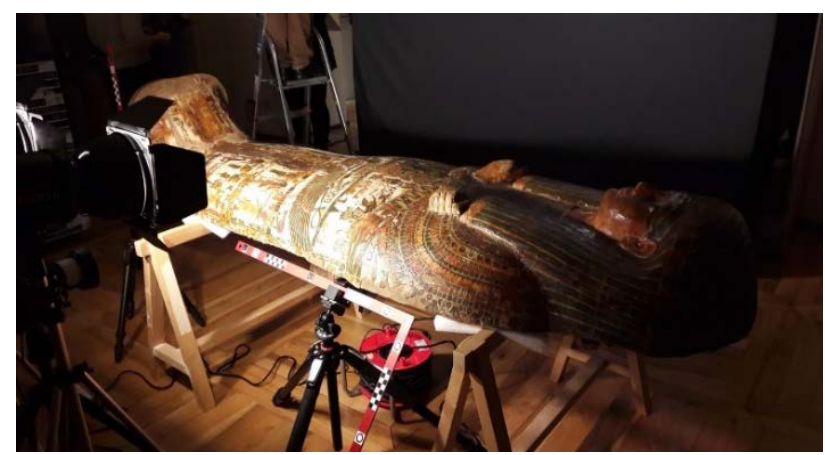

Figure 2. The coffin during the photogrammetric acquisition.

On the contrary, the public had the possibility to glimpse the entire process of the acquisition via scanner, as this operation was performed along the exhibition path of the Museum, on a cordoned-off area in order to avoid vibrations (Figure 3). Visitors stopped to ask questions and to follow "live" the progressive construction of the three-dimensional model on screen.

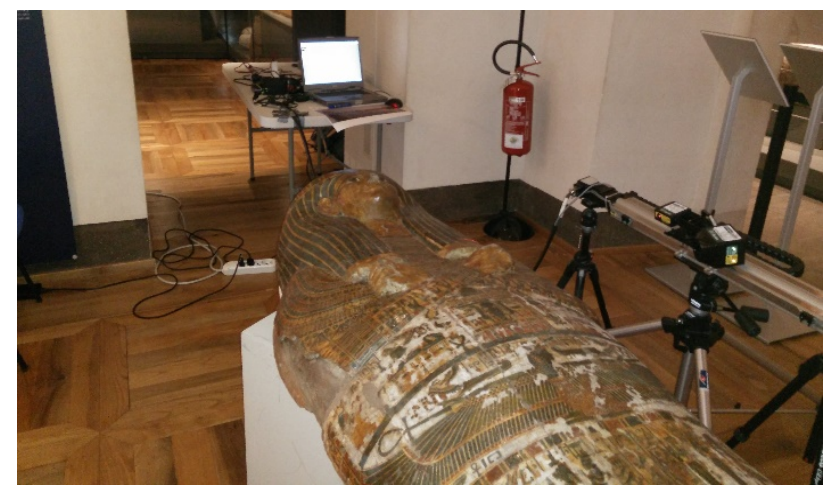

Figure 3. The coffin during the scanner acquisition.

The following discussion will consider only the lid of the coffin, even if the external surfaces of both the two parts were acquired.

3.1.1 Triangulation Scanner Survey: the scanner employed was the ShapeGrabber, which is composed by a tripod, a $600 \mathrm{~mm}$ slider and a scanner head with a laser and a camera. It is possible to switch between two different heads according to the desired resolution. The SG102 head with a maximum nominal resolution of $0,01 \mathrm{~mm}$ and the SG1002 head with a maximum nominal resolution of $0,1 \mathrm{~mm}$ were available (Figure 4 , Table 1 ). The head moves along the motorized slider that allows to increase the yField-Of-View (FOV), reducing the number of stations and so speeding up the survey phase.

The choice of the head affects the field, the depth of view, the time for each scan and the number of stations (higher resolution means higher time and number of stations). In the specific case, the dimension and the configuration of the slider prevented the possibility to use the SG102 head, as the required depth of view implied getting dangerously close to the coffin. 


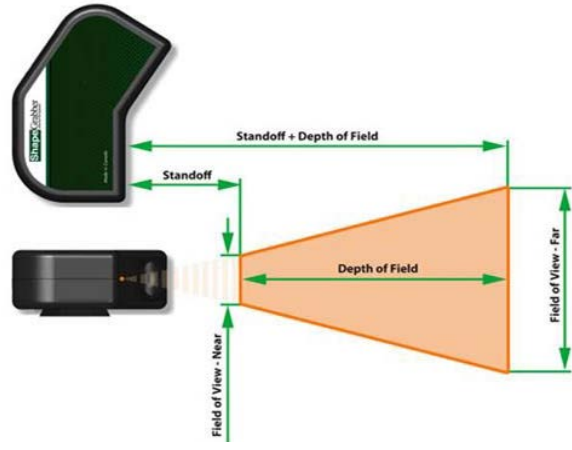

Figure 4. ShapeGrapper head parameters.

Furthermore, there would have been two other key problems with the use of the SG102 head: i) the speed of points acquisition would have increased exponentially in order to achieve a 0,01 mm point-to-point space; ii) the SG102 has a very small x-FOV (85 $\mathrm{mm}$ near and $175 \mathrm{~mm}$ far) which would have considerably increased the necessary number of scans related to the geometric dimension of the coffin. In addition, the high number of high range maps would have been difficult to manage during the alignment phase. Finally, considering also that a $0,01 \mathrm{~mm}$ resolution was not required and that the acquisition and processing times would have been too time-consuming, the SG102 was excluded and instead the SG1002 was chosen.

\begin{tabular}{|c|c|}
\hline \multicolumn{2}{|c|}{ SG1002 } \\
\hline \multicolumn{2}{|c|}{$X$ Field Of View } \\
\hline Near & $200 \mathrm{~mm}$ \\
\hline Far & $640 \mathrm{~mm}$ \\
\hline Point spacing (mid) & $320 \mu \mathrm{m}$ \\
\hline \multicolumn{2}{|c|}{ Y point spacing in scan direction } \\
\hline \multicolumn{2}{|c|}{$\begin{array}{l}\text { Function of scan velocity. On a typical ShapeGrabber system } \\
\text { minimum point spacing is } 10 \mu \mathrm{m}\end{array}$} \\
\hline \multicolumn{2}{|c|}{ Z range } \\
\hline Standoff & $295 \mathrm{~mm}$ \\
\hline Depth of field & $800 \mathrm{~mm}$ \\
\hline Resolution (mid) & $25 \mu \mathrm{m}$ \\
\hline $1 \sigma$ noise (mid) & $90 \mu \mathrm{m}$ \\
\hline \multicolumn{2}{|c|}{ Dimensions } \\
\hline Length & $295 \mathrm{~mm}$ \\
\hline Width & $75 \mathrm{~mm}$ \\
\hline Height & $141 \mathrm{~mm}$ \\
\hline Weight & $1,14 \mathrm{~kg}$ \\
\hline \multicolumn{2}{|c|}{ Data rate } \\
\hline Minimum & $18.000 \mathrm{pts} / \mathrm{s}$ \\
\hline Maximum & $100.000+\mathrm{pts} / \mathrm{s}$ \\
\hline
\end{tabular}

Table 1. SG1002 technical data.

The head has a depth of field of $505 \mathrm{~mm}$ (it records data between $295 \mathrm{~mm}$ and $800 \mathrm{~mm}$ measured from the laser emitter). The medium resolution in this direction $(\mathrm{Z})$ is equal to $0,025 \mathrm{~mm}$. The field of view (X direction) spans from $200 \mathrm{~mm}$ to $640 \mathrm{~mm}$ depending on the distance from the object, the space between points is $0,32 \mathrm{~mm}$. The point spacing in $\mathrm{Y}$ direction is a function of the head velocity, at the lowest speed is equal to $0,01 \mathrm{~mm}$.

The head of the scanner is remotely controlled via the proprietary software of the scanner. The controllable parameters are i) the velocity of the head along the tray, ii) the power of the laser beam and iii) the length of the horizontal movement. The first parameter affects the precision of the survey; higher speed means lower precision. By playing with the second parameter, it is possible to reduce the noise in clearly visible areas, lowering the power of the laser. On the other hand, if there are dark-coloured areas, it is necessary to increase the power of the beam, otherwise the laser would be absorbed by the object. We decided to use only the central part of the slider, within a range between $+200 \mathrm{~mm}$ and $-200 \mathrm{~mm}$ from the head of the tripod, because this is the calibrated area where the technical specification is ensured. This decision allows also avoiding unwanted vibrations or movements of the system along the portion at the end of the slider during the acquisition. Before performing the survey, the instrument was tested on a planar surface (Figure 5), in the configuration: resolution $0,1 \mathrm{~mm}$, laser power 4 , distance from the tested surface $500 \mathrm{~mm}$. The test aims to verify the technical data given by the manufacturer.

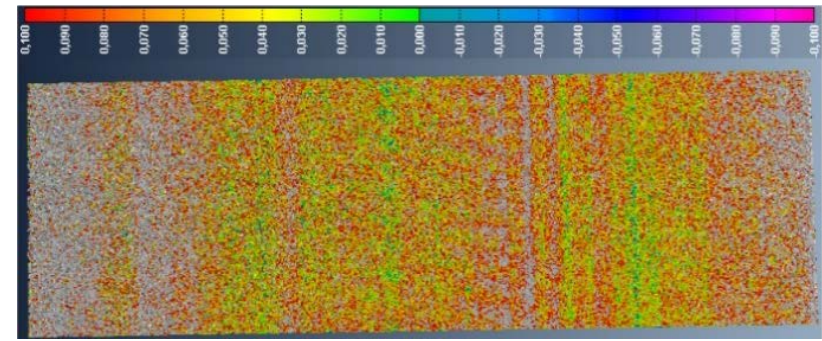

Figure 5. The image shows the distance between the scan of a planar surface and the interpolated model of the planar surface.

On the image, the threshold for the comparison is set $\pm 0,1 \mathrm{~mm}$. The red points highlight the noise of the scan in the range set. In grey the points with an offset between the scan and the ideal plane greater than $0,1 \mathrm{~mm}$, which cover the areas at the end of the range map. As previously mentioned, we did not allow the head to reach the ends of the slide, in order to exclude from the data acquisition these un-calibrated areas, subject to vibrations. After these considerations, the survey followed this pipeline: i) calibration of the scanner head using a calibrated plate; ii) acquisition; iii) scans cleaning to remove noise, outliers, and sparse points on the edge of the object, keeping only the best data for each scan station; iv) data alignment using PolyWorks; v) merge of the scans into the final three-dimensional polygonal model. The acquisition followed a seven strips scheme running from the head to the feet with an overlap of about $60 \%$ giving as a result 81 scans. The work was organised to obtain the final model in the least amount of time to prepare the model that had to be 3D printed for the forthcoming exhibition. While an operator was performing the survey, a second one was prealigning the data and suggesting which part had to be covered with further scans. At the end of the third day, before leaving the Museum, the elaboration of the mesh model was already in progress. Before the meshing stage, the range images were cleaned and properly aligned (Figure 6) using the ICP (Iterative Closest Point) algorithm and reaching a mean standard deviation of $0,086 \mathrm{~mm}$ thanks to the good overlap among the scans.

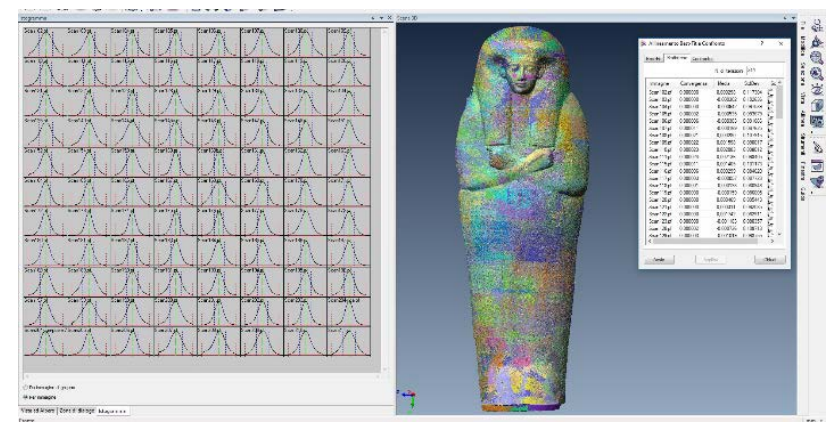

Figure 6. The 81 scans aligned. 
In a first phase, all scans of the lower ring of the coffin were registered in series, subsequently blocking those already aligned. Once the entire lower ring was closed, all scans were unlocked and the alignment was optimized. The other acquisitions were recorded using this data block as a reference.

Once this first step of the registration process, was completed, i) all the individual scans were cleaned eliminating the edges with low quality of the points, ii) all the scans were unlocked and iii) an ICP process was launched for the optimization of the final alignment. The sum of all the scans gave as a result a discrete 3D model composed of about 180.000 .000 points (Figure 7).

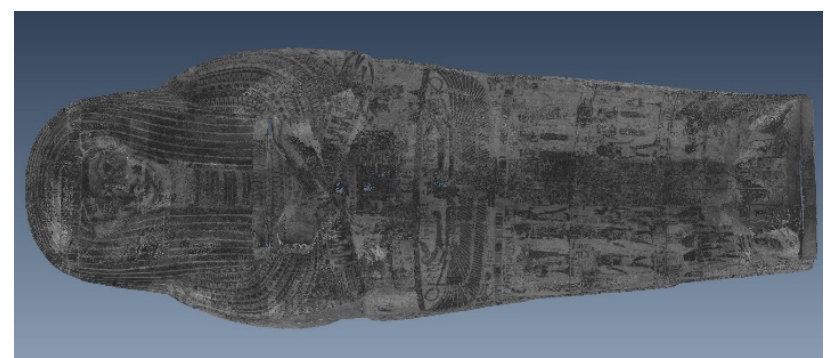

Figure 7. The discrete 3D model, in grey scale the point's reflectance is represented.

Theoretically, it is possible to build a mesh of about 90 million triangles. Considering that, the external surface of the coffin measures about $2 \mathrm{~m}^{2}$, the edge length of the triangles on average should be equal to $0,2 \mathrm{~mm}$ :

$$
\begin{aligned}
& \text { triangle area }=\frac{\text { lid area }}{\mathrm{n}^{\circ} \text { of triangles }} \\
& \text { triangle area }=\frac{2 * 10^{6} \mathrm{~mm}^{2}}{90.000 .000} \\
& \text { triangle area }=0,25 \mathrm{~mm}^{2} \\
& \text { triangle edge }=\sqrt[2]{\frac{4}{\sqrt[2]{3}} * \text { triangle area }} \\
& \text { triangle edge }=\sqrt[2]{\frac{4}{\sqrt[2]{3}} * 0,25 \mathrm{~mm}^{2}} \\
& \text { triangle edge }=0,23 \mathrm{~mm}
\end{aligned}
$$

The equation (1) allows to calculate the area of each triangle of the theoretical mesh obtained by interpolating every point of the discrete 3D model. The second equation (2) returns the value of the edge length of an equilateral triangle knowing the area of the triangle. The result obtained is consistent with the instrument specifications and the settings imposed for the survey. Moreover, this value is the same obtained in the Pietà Rondanini case of study which aim was to obtain a 1:1 digital model of the statue. As it is not easy, obviously, to handle a 90 million triangles model, PolyWorks decimation algorithms were exploited to calculate an optimized mesh of the coffin. The parameters imposed during the meshing stage were:

- $\quad$ maximum distance $=1 \mathrm{~mm}$

- $\quad$ sampling step $=0,2 \mathrm{~mm}$

- $\quad$ standard deviation $=0,1 \mathrm{~mm}$

In the modelling philosophy of PolyWorks, there must be a minimum amount of overlap between images in a set of 3D images. The maximum distance parameter defines an acceptable maximum distance between a 3D image point and another 3D image. The maximum distance parameter thus acts as a threshold used in detecting overlaps in the set of $3 \mathrm{D}$ images. This detection is essential in order to reconstruct a non-redundant surface representation where each part of the measured object is only described once. The value should be sufficiently large to detect all actual surface overlaps. The density of the mesh is determined by the surface sampling step, which defines the mean edge length of the triangles of the interpolated model.

The standard deviation value is obtained from the input alignment project if a best-fit alignment operation was performed.

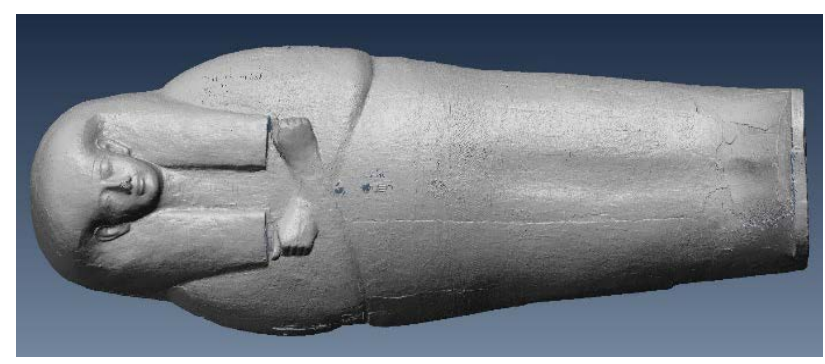

Figure 8. The mesh model.
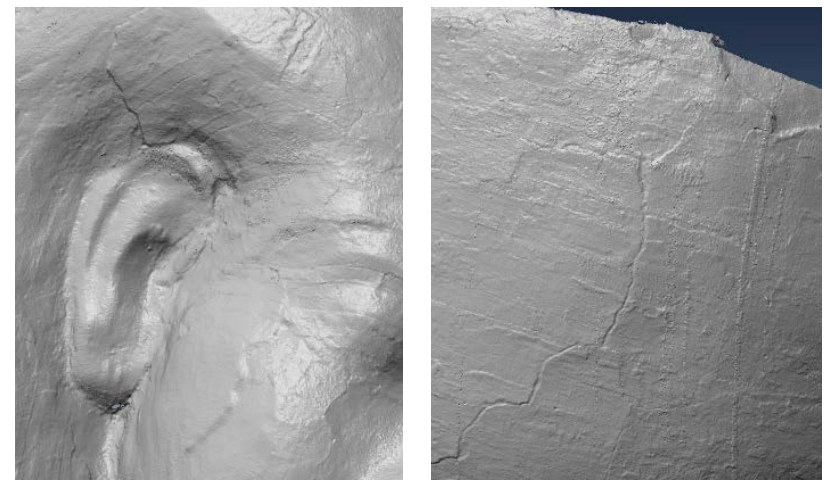

Figure 9. Two details of the mesh model.

$0,1 \mathrm{~mm}$ is the rounded up of the alignment value obtained in the alignment stage: it was equal to $0,086 \mathrm{~mm}$. If an alignment operation was not performed, the default value 0,05 is proposed. It is possible to replace this value with the standard deviation of the digitizer $(\sigma)$ that captured the scans, again $0,1 \mathrm{~mm}$ (rounded up of $0,09 \mathrm{~mm}$ ). After the cleaning and repairing stage of the mesh, the result is a final, lightweight, and easy to handle 3D model (Figures 8 and 9 ) of around 8 million triangles.

3.1.2 Photogrammetric Survey: The photogrammetric acquisition was performed using a full-frame Nikon D810 camera. The images were taken in raw mode at the maximum resolution (7360 pixels * 4912 pixels) using a tilt\&shift $45 \mathrm{~mm}$ lens (no tilt nor shift applied) in manual fixed focus. The camera was mounted on a tripod with a micrometric positioning sliding plate to check the focus depth around the object without changes the principal distance.

The mean GSD of the photogrammetric survey is less than 0,1 $\mathrm{mm}$ since the sensor pixel pitch is $4,88 \mu \mathrm{m}$ and the distance of acquisition from the object was about $70 \mathrm{~cm}$. The acquisition design is a wraparound free network with images as much as possible nadir to the surface. Around the corners and the edges, the geometry is semi-circular with steps of 10 degrees. This network design guarantees a longitudinal and transversal overlap of about $80 \%$ between two adjacent images (Figure 10). The correctness of the colour of the images is guaranteed by the use of a colour checker palette. 


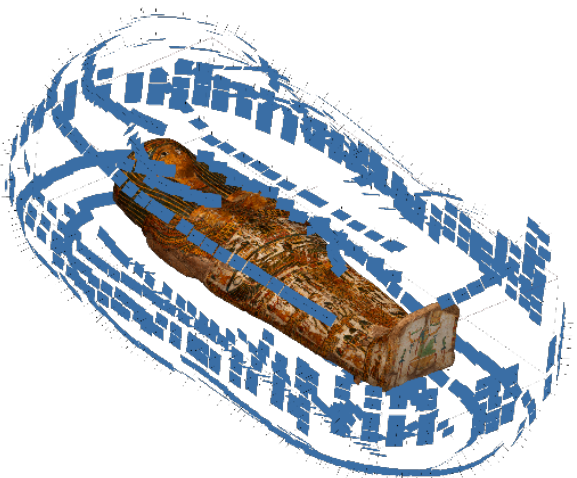

Figure 10. Wraparound network.

Four pre-calibrated L-shaped bars were placed in different positions during the whole survey (i.e. they were next to the coffin head during the acquisition of the head, then moved on a side and finally placed next to the feet). In this way, it was possible to accurately scale the reconstruction and to check for deformations in several parts of the coffin.

The bars were designed to use black and white non-coded centroids and geometrical patterns as targets. The calibration phase took place in the 3DSurveyGroup Lab of Politecnico di Milano using as reference the Brunson 803-MCP kit (Figure 11). The Invar tubing in the 803-MCP is manufactured to its stated length within $\pm 0,003 \mathrm{~mm}$. In addition, the Invar tubing components are handpicked for each kit so that the total stack up error does not exceed $0,013 \mathrm{~mm}$. This means that the sum of the all tubes connected together end-to-end will maintain the total length within the stack up error.

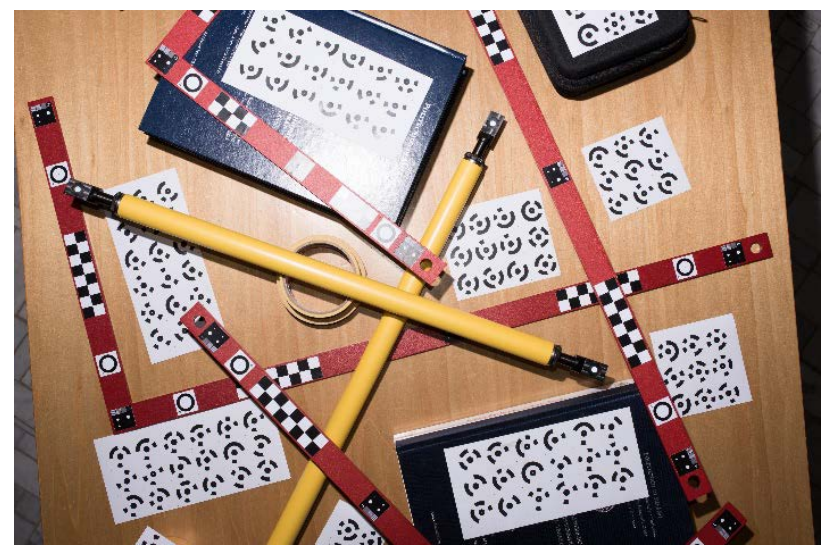

Figure 11. Bar calibration.

The camera placed on a photographic tripod and the use of a remote shutter made sure to avoid vibrations as much as possible that, together with a fast shutter speed, guaranteed sharp images. Next to the tripod, there were two external flashlights (Godox QS600II) synchronized with the camera. As known, (Conen et al., 2018, Menna et al., 2016) the photogrammetric matching of reflective surfaces may result in incorrect measurements, blunders, and noise in the final point cloud. To solve the problem of cross-polarisation can be used: polarizing filters can be mounted both on the camera lens and on the light source. Cross polarization is achieved when light is polarized twice, at the emitting source in one direction and after having reflected off the object surface before entering the camera in the opposite direction. The photogrammetric set up used for this acquisition employed two studio flashlights to illuminate the subject; both flashlights had been polarized in the same direction (vertical polarisation) by superimposing two fixed linear polarizers sheet onto the lamp hoods. A circular polarizer was attached to the lens and positioned to polarize the light in the horizontal direction. This photographic technique allowed to potentially remove all of the reflections and consequently eliminating the main cause of noise during the dense matching reconstruction phase. To ensure the best result possible with the cross-polarisation it is mandatory to control each light source so that the polarized flashlights are the only source of illumination for the scene. The polarisation direction of the two studio torches should be parallel to one another. Moreover, for the best possible control, the camera circular polarizer can be manually adjusted, image by image, each time the set-up moves; the results must be checked immediately. The acquisition of the lid of the coffin lasted one day, during which time the operator acquired 818 photos (Table 2).

\begin{tabular}{|l|r|}
\cline { 2 - 2 } \multicolumn{1}{c|}{} & \multicolumn{1}{c|}{ Coffin lid } \\
\hline Number of photos & 818 \\
\hline Distance of acquisition & $0,7 \mathrm{~m}$ \\
\hline GSD & $0,08 \mathrm{~mm} /$ pixel \\
\hline Re-projection error on control points & 0,6 pixel \\
\hline$\sigma$ & $0,07 \mathrm{~mm}$ \\
\hline Dense matching $n^{\circ}$ of points & $\sim 400 \mathrm{million}$ \\
\hline$N^{\circ}$ of mesh triangles & 30 million \\
\hline Mean edge length & $0,5 \mathrm{~mm}$ \\
\hline Ortophoto resolution & $0,06 \mathrm{~mm} /$ pixel \\
\hline
\end{tabular}

Table 2. Photogrammetric data.

After the acquisition the workflow of the photogrammetric elaboration implied the following steps: i) photo-alignment, computation of internal and external parameters; ii) manual checking and filtering of the tie-points; iii) scaling of the 3D reconstruction; iv) dense cloud reconstruction and data cleaning; v) mesh model (Figure 12); vi) texturing; vii) high-resolution orthophoto.
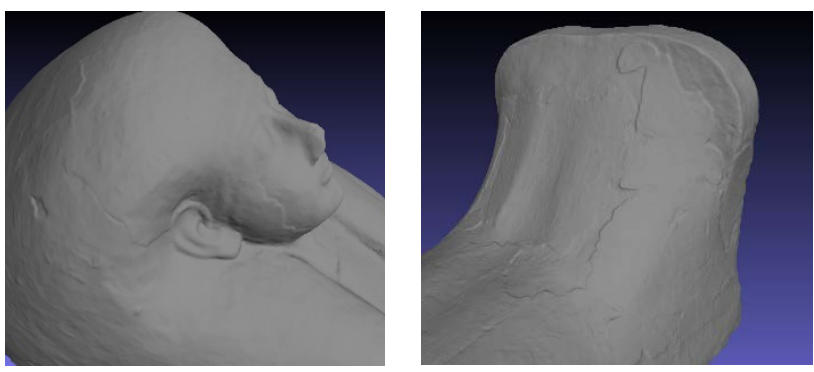

Figure 12. Two details of the photogrammetric model.

Agisoft Photoscan was used to follow the photogrammetric pipeline. The alignment was computed using the image at full resolution. The dense matching reconstruction was performed with the "high" quality, this implies the downscaling of the preliminary image size by a factor of 4 (2 times by each side). At the end of the workflow, the dense point cloud was composed of nearly 400 million points, and the mesh by 30 million triangles.

\section{GENERAL ISSUES}

Some issues arose during the acquisition and elaboration processes. First, the object itself, due to its fragility, uniqueness and cultural value, could not be placed in all the position that would have been more convenient from the perspective of the 
survey; this happened, in particular, during the scanner acquisition. In fact, with the ShapeGrapper it was not possible to record the inner portion of both pieces of the coffin, because of the rigidity of the system tripod-scanner-head and the impossibility to place the pieces on a side.

In other contexts, the survey of the whole coffin could have been tried, but it would have required a very long time because of the high number of stations and the slowness of the instrument. In the three days of survey, the scanner operator acquired only the outer part of the coffin; while with photogrammetry, it was possible to record the entire object, inside and outside.

Moreover, the ratio object-dimensions / scanner-resolution is too high, i.e. the desired final resolution is quite high on an object of such a size. During the elaboration, it was necessary to exclude seven scans that presented small deformations, due to micromovements of the tripod; because of what just stated in the previous sentence, this was not an issue. Other problems occurred in the registration phase caused by the redundancy of data and the sharp angle between the laser beam and the coffin (Peng et al, 2011).

The photogrammetric method it turned out to be very flexible in the acquisition phase that allowed the survey even of the hidden internal parts without particular problems. The most significant difficulties in the acquisition phase are related to the required photogrammetric quality of the shots: images must be entirely in focus while using a fixed blocked focal length and therefore, without the possibility of focusing during acquisition. The photographic quality is also very important, as images must be contrasted, chromatically uniform and without reflections, or micro-reflections. All these requirements involve the difficult photographic acquisition, especially when the expected resolution is sub-millimetric, and the acquisition distance is minimal.

The main issues are related to the matching time if the full resolution is required. In this case, the elaboration time take so long that the method results not compatible with the allowed times. For this reason, the model was created performing the down sampling from the initial image resolution. In the next paragraph, a comparison has been made to understand the grade of simplification obtained making this choice.

\section{COMPARISON}

The photogrammetric model obtained using the full resolution of the images with the scanner model have been compared, in some little test areas and using a limited number of images. This comparison is intended to investigate the smallest details on the coffin, such as the thickness of the paint on the wooden surface. One of the test areas chosen for the comparison corresponds to the "left leg" of the coffin (Figure 13). This area presents different kinds of small details: a crack in the wood and different layers of painting placed on a significant curvature.
As clearly visible, the geometries coming from the scanner are highly defined. It is possible to distinguish the shape of hieroglyphics painted on the coffin and the sharp contours of the edges. The point cloud obtained processing the images with high quality is not defined enough to show all the details and the border of cracks and discontinuities results smoothed. Finally, the point cloud computed using the full resolution of the images presents more sharp edges closer to the scanner geometry even if the hieroglyphics are not enough clearly modelled.

Three different elaborations of the same surface will be compared with the one taken as reference, the result of the scanner: i) the mesh obtained from the photogrammetric point cloud using the "high" quality (i.e. the quality used for the whole coffin); ii) the mesh obtained directly form the depth maps calculated using the "high" quality; and iii) the mesh obtained directly form the depth maps calculated using the "ultra-high" quality (Figure 14).

This is a double comparison: the first one regards the quality of the results on fine details, generating the mesh from the point cloud or directly from the depth maps.

The second comparison is about the accuracy (quantitative comparison) of the mesh obtained using the full resolution of the images or down sampling them by $1 / 4$.

The qualitative comparison, as expected, shows that the mesh obtained directly from the depth maps is less noisy than the one built starting from the dense matching data. This happens because of the slight noise of the point cloud due to different factors, such as the redundancy of the photos or the remaining reflections passed through the polarizer. Moreover, the developers of the software give some hints on using one or the other meshing method. Mesh from depth maps setting allows to use all the information from the input images more effectively and is less resource demanding compared to the dense cloud based reconstruction. Consequently, the quantitative evaluation will consider only the meshes built starting from the depth maps.

The comparison is performed with Geomagic Design X, the two photogrammetric meshes are aligned with the best-fit algorithm to the scanner one, and then the results are evaluated using the mesh deviation tool (Figure 15).

It is clear that the mesh obtained using the full resolution of the images is much more detailed than the first one. The comparison highlights two differences between the meshes. The former regards the number of triangles higher than the threshold equal to $\pm 0,2 \mathrm{~mm}$, the latter is about global accuracy (Table 3).

\begin{tabular}{|l|c|c|c|c|}
\hline & $\begin{array}{c}N^{\circ} \text { of } \\
\text { images/ } \\
\text { scans }\end{array}$ & $N^{\circ}$ of points & $\begin{array}{c}N^{\circ} \text { of } \\
\text { triangles }\end{array}$ & Time \\
\hline Scanner model & 4 & 22.630 .625 & 1.009 .007 & $/$ \\
\hline Mesh from p.c. (high) & 15 & 13.640 .731 & 33.277 .703 & $11^{\prime} 25^{\prime \prime}$ \\
\hline Mesh from d.m. (high) & 15 & $/$ & 33.863 .538 & $10^{\prime} 7^{\prime \prime}$ \\
\hline Mesh from d.m. (ultra-high) & 15 & $/$ & 132.584 .964 & $50^{\prime} 15^{\prime \prime}$ \\
\hline
\end{tabular}

Table 3. Summary of the comparison. Point cloud (p.c.); Depth maps (d.m.)
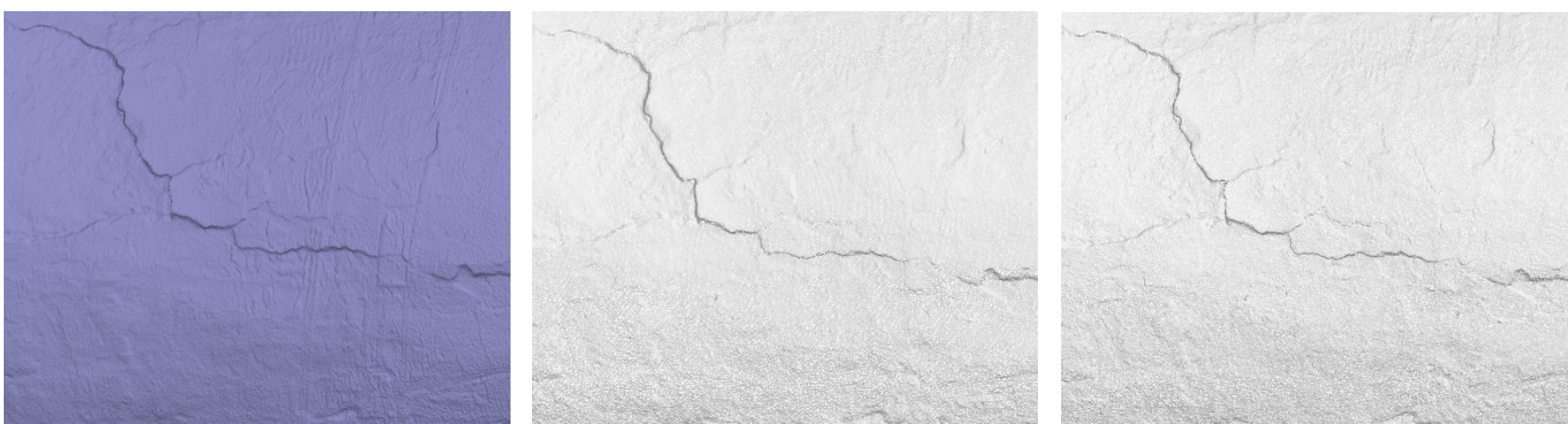

Figure 13. On the left, the mesh from the scanner survey. In the centre, The photogrammetric point cloud in high quality. On the right, the photogrammetric point cloud in ultra-high quality. 

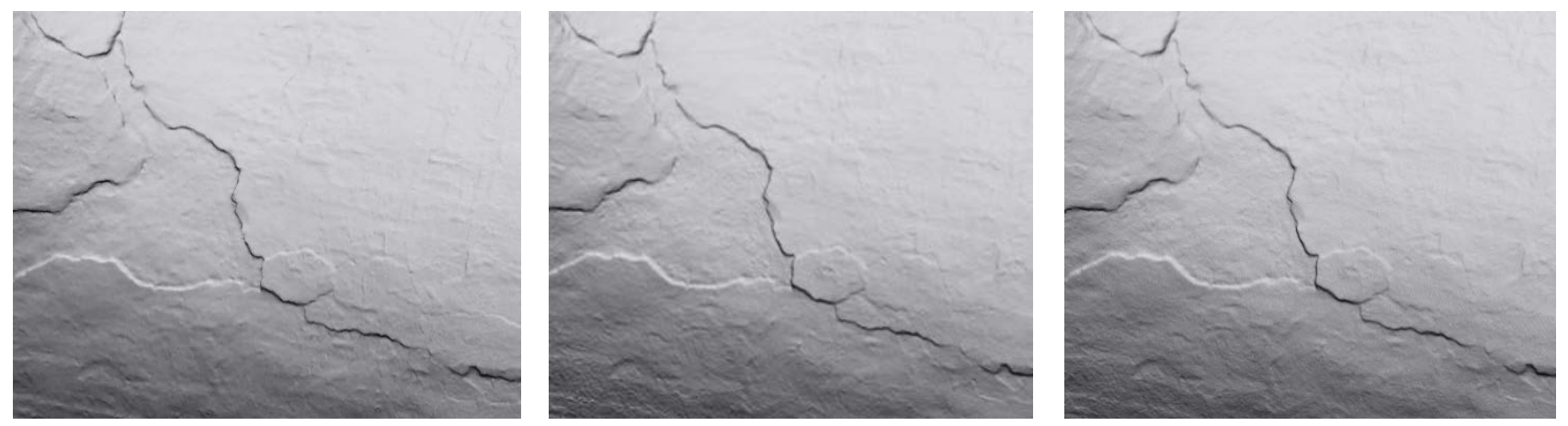

Figure 14. On the left, detail of the scanner model. In the centre, detail of the photogrammetric model from depth maps in high quality. On the right, detail of the photogrammetric model from depth maps in ultra-high quality.
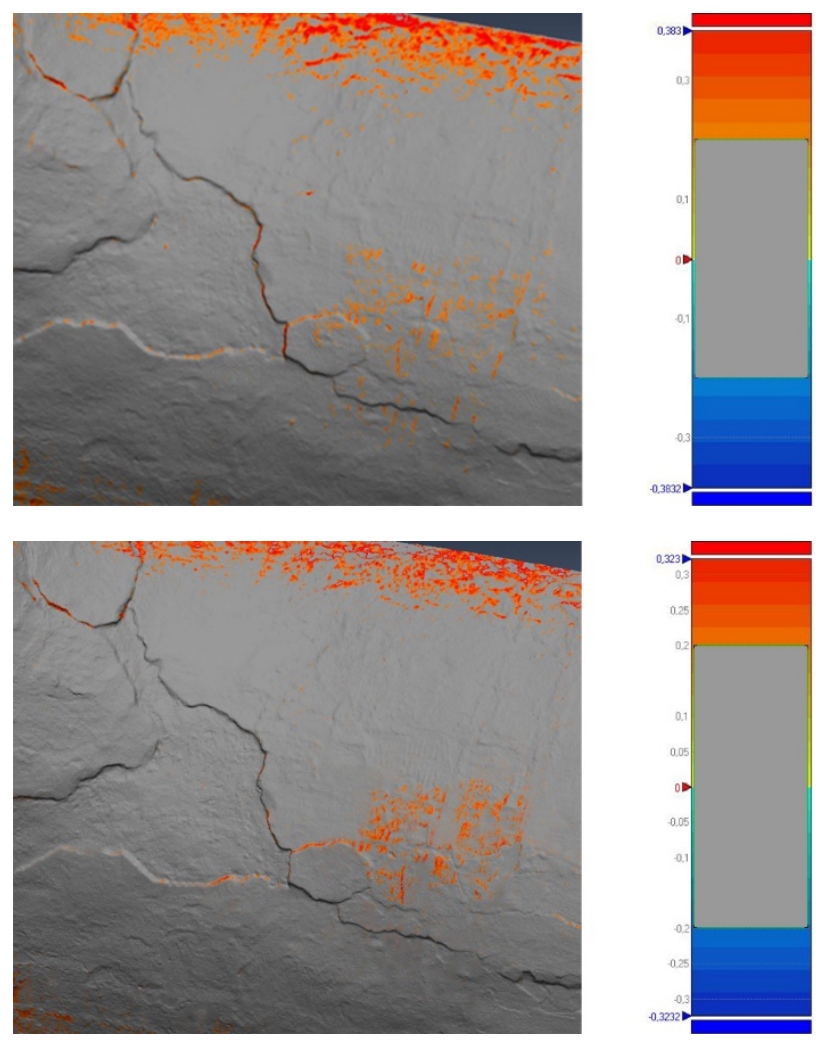

Figure 15. Up, the mesh distances between scanner model and the high resolution mesh from depth maps. Down, the mesh distances between scanner model and the ultra-high resolution mesh from depth maps.

The orange/red areas in the second image are less than in the first one, so that, there are fewer parts above the threshold. Moreover, observing the values of the colored bars. In the first case, the farthest triangles from the reference scanner mesh are close to 0,4 $\mathrm{mm}$. On the other hand, the second bar demonstrates that the values of the farthest triangles tend to $0,3 \mathrm{~mm}$. These tests suggest that with the photogrammetry it is possible to reach the same resolution and accuracy of the triangulation scanners (Rizzi et al., 2007), but the time required to get such results is quite high. Moreover, it is necessary to use a coherent number of images; too many images cause noisy and redundant data, few images cause lack of data. The images must be as much as possible sharp from the photographic point of view and the elaboration must consider the photos at the original size.

\section{CONCLUSION}

Experimentation has shown that both methodologies lead to the expected result, albeit in different ways, presenting some advantages and disadvantages that validate and consolidate the initial expectations.

The main pros of the scanner approach are: i) a very high measurement accuracy and resolution that does not depend on external factors and ii) real-time modelling. The main cons are: i) the lack of good quality of colour information, ii) the lack of a reference to check the final accuracy iii) the low flexibility in managing the scanner during the acquisition and iv) the high costs of the instrumentation.

On the contrary, photogrammetry is: i) an economically convenient method, ii) very flexible during the acquisition, iii) allows geometric reconstruction together with metric colour reprojection and iv) allows a statistical check of the accuracy of every point of the final model. The cons are i) the long processing time to obtain the full resolution model and ii) the highperformance computation devices requested to perform the elaborations.

It is clear that many of the advantages of one method would solve the problems of the other. This testifies how, even today, for the types of works required in the world of Cultural Heritage, the integration of the two methods is necessary to obtain a complete and exhaustive survey. It is evident that the scanner equipment can provide a more resolute and accurate geometric result with tight deadlines, but at very high costs, while, on the other hand, photogrammetry comes very close to the same results, but requests a long elaboration process, a cost in itself, that must be considered.

To survey a coffin such as that of Butehamon, the choice of one or the other method is only dependent on the final purpose. For communication purposes, the high-resolution is not indispensable, and might indeed counterproductive. It is therefore clear that the photogrammetric way is optimal for this purpose because it is economical, flexible, fast and provides the colour component (Figure 16). If instead, the resolution and the accuracy are the main goals for metrological study purposes at a microscopic level or for special purposes as for example, 1:1 3D printing, the scanner is the tool that provides the best results.

As a final, tangible output of the whole process and of the various threads that can spring from these operations, the survey data were enhanced realizing a micro-mapping installation, which combines the model coming from the scanner as physical, 3Dprinted support, with the texture derived from the photogrammetry. This multimedia product is on display at the very end of the exhibition Archeologia Invisibile (Invisible Archaeology) at Museo Egizio of Turin from March 2019 to January 2020 (Greco, 2019). 


\section{ACKNOWLEDGMENTS}

The authors would like to thank Alessia Amenta, Musei Vaticani, the staff of Museo Egizio for their support, and 3DOM and Fabio Remondino for lending the scanner. Special thanks to Fabio Menna and Erica Nocerino for the valuable advice on crosspolarization methods.

\section{REFERENCES}

Barsanti, S.G., Remondino, F., Fenández-Palacios, B.J., Visintini, D., 2014. Critical factors and guidelines for 3D surveying and modelling in cultural heritage. In: International Journal of Heritage in the Digital Era, Vol. 3(1), pp. 141-158, doi.org/10.1260/2047-4970.3.1.141.

Conen, N., Hastedt, H., Kahmen, O., Luhmann, T., 2018. Improving image matching by reducing surface reflections using polarising filter techniques. In: The International Archives of the Photogrammetry Remote Sensing and Spatial Information Sciences, Vol. XLII-2, pp. 267-274, doi.org/10.5194/isprsarchives-XLII-2-267-2018.

Fischnaller, F., Guidazzoli, A., Imboden, S., De Luca, D., Liguori, M.C., Russo, A., Cosentino, R., De Lucia, M.A., 2015. Sarcophagus of the Spouses installation intersection across archaeology, 3D video mapping, holographic techniques combined with immersive narrative environments and scenography. In: Digital Heritage, pp. 365-368, doi.org/10.1109/DigitalHeritage.2015.7413903.

Gomes, L., Silva, L., and Bellon, O., 2014. 3D reconstruction methods for digital preservation of cultural heritage: a survey. In: Pattern Recognition Letters, Vol. 50, pp. 3-14, doi.org/10.1016/j.patrec.2014.03.023.

Greco, C., 2019: The biography of objects, 2019. In: The International Archives of the Photogrammetry Remote Sensing and Spatial Information Sciences, Vol. XLII-2/W11, pp. 5-10, doi.org/10.5194/isprs-archives-XLII-2-W11-5-2019.

Guidi G., Beraldin J., Atzeni C., 2004. High-accuracy 3D modeling of cultural heritage: the digitizing of Donatello's “Maddalena”. In: IEEE Transactions on Image Processing, Vol. 13 (3), pp. 370-380, doi.org/10.1109/TIP.2003.822592.

Mandelli, A., Achille, C., Tommasi, C., Fassi, F., 2017. Integration of 3D models and diagnostic analyses through a conservation-oriented information system. In: The International Archives of the Photogrammetry Remote Sensing and Spatial Information Sciences, Vol. XLII-2/W5, pp. 497-504, doi.org/10.5194/isprs-archives-XLII-2-W5-497-2017.
Menna, F., Nocerino, E., Remondino, F., Dellepiane, M., Callieri, M., Scopigno, R., 2016. 3D digitization of an heritage masterpiece - a critical analysis on quality assessment. In: The International Archives of the Photogrammetry Remote Sensing and Spatial Information Sciences, Vol. XLI-B5, pp. 675-683, doi.org/10.5194/isprs-archives-XLI-B5-675-2016.

Peng, Q., Sanchez, H., 2011. 3D digitizing technology in product reverse design. In: Proceedings of the Canadian Engineering Education Association, doi.org/10.24908/pceea.v0i0.3966.

Pontin, M., Pirotti, F., Girardi, S., Voltolini, F., 2005. Dati RGB e laser scanner per la modellazione di piccoli oggetti complessi. In: Atti Asita.

Prestipino G., Santamaria U., Morresi F., Amenta A., Greco C., 2015. Sperimentazione di adesivi e consolidanti per il restauro di manufatti lignei policromi egizi. In: Atti XIII Congresso Nazionale IGIIC - Lo Stato dell'Arte, Centro di Conservazione e Restauro La Venaria, Torino, 22-24 ottobre 2015, pp. 261-270.

Rizzi, A., Voltolini, F., Remondino, F., Girardi, S., Gonzo, L., 2007. Optical measurement techniques for the digital preservation, documentation and analysis of cultural heritage. In: Optical 3-D measurements techniques VIII, Vol. 2, pp. 16-24, Zurich.

Rossi, C. 2019. Aristotle's mirror: combining digital and material culture. In: The International Archives of the Photogrammetry Remote Sensing and Spatial Information Sciences, Vol. XLII2/W11, pp. 1025-1029, doi.org/10.5194/isprs-archives-XLII-2W11-1025-2019.

Taylor, J. M., Godin, G., Beraldin, J.-A., 2002. Heritage recording applications of high resolution 3D imaging. In: Proc. $16^{\text {th }}$ International Conference on Pattern Recognition, Vol. 2, pp. 520, doi.org/10.1109/ICPR.2002.1048352.

Tucci, G., Bonora, V., Conti, A., Fiorini, L. 2017. High-quality $3 \mathrm{D}$ models and their use in a cultural heritage conservation project. In: The International Archives of the Photogrammetry Remote Sensing and Spatial Information Sciences, Vol. XLII2/W5, pp. 687-693, doi.org/10.5194/isprs-archives-XLII-2-W5687-2017.

http://www.museivaticani.va/content/museivaticani/it/collezioni /musei/museo-gregoriano-egizio/museo-gregorianoegizio/progetti-scientifici/vatican-coffin-project/mostrarestauro-di-butehamon.detailmenu.html (12/06/2019)

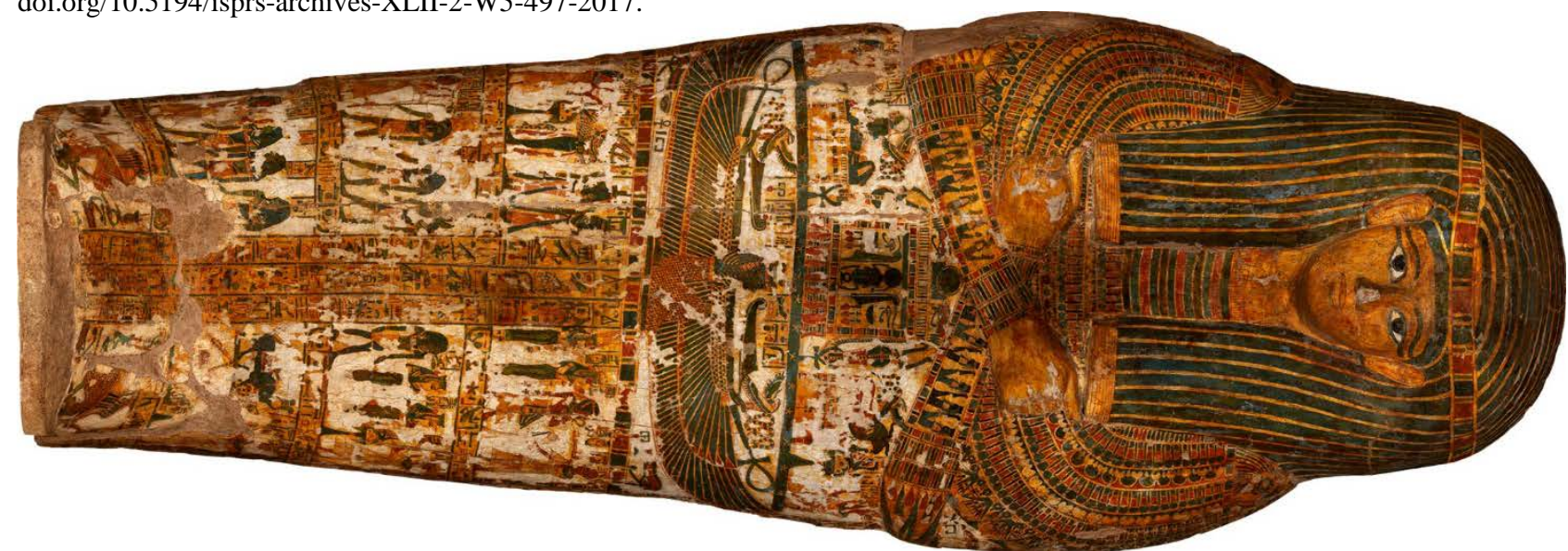

Figure 16. High-resolution orthophoto of the coffin. 